\title{
La orientación en los hombres. Acerca del problema de la antropología
}

\author{
Orientation towards Individual Persons. On the \\ Problem of Anthropology ${ }^{l}$
}

Werner Stegmaier ${ }^{2}$

Greifswald Universität, Alemania

Recepción: 27 de octubre del 2020

Evaluación: 27 de noviembre del 2020

Aceptación: 30 de noviembre del 2020

Traducción del alemán por Adrián Muñoz, Bauhaus-Universität Weimar, Alemania.

2 Realizó su trabajo de habilitación en la Rheinische Friedrich-Wilhelms-Universität (Bonn). Doctor por la Eberhard Karls Universität Tübingen y estudios de Filosofía, Germanística y Latinística en la Eberhard Karls Universität Tübingen y en la Universität Wien.

Correo electrónico: stegmai.pr@gmail.com

Stegmaier, W. (2020). La orientación en los hombres. Acerca del problema de la antropología. Cuestiones de Filosofía, 6 (27), 127-156. 


\section{Resumen}

La antropología padece el dilema de hablar del hombre en general, aunque siempre se tiene que tratar, sin embargo, con el hombre particular. Ella surge de aquello que Nietzsche denomina la 'muerte de Dios': cuando Feuerbach sustituye la teología por la antropología, cuida la imagen del hombre de tal manera que éste se convierte en ideal y norma. De esto buscan apartarse Darwin, Nietzsche, Freud, y también parte de la antropología filosófica del siglo 20: desde la perspectiva de la evolución, el animal humano es un constante experimento que a lo largo del tiempo trasciende todo intento de definición esencial e intemporal. Un siglo después de Nietzsche, Luhmann desidealiza y desnormativiza 'al' hombre: descompone la aparente unidad del hombre en tres sistemas -físico, psíquico y de comunicación de la sociedad, que sin determinarse uno al otro, se examinan y reaccionan mutuamente. Los márgenes que permanecen en la relación de los sistemas, que son entorno de uno para el otro, pueden ser concebidos de la mejor manera a través del concepto de 'orientacion': todo lo que se orienta, sea el cuerpo, la conciencia o la sociedad, es un sistema de observación que se orienta en su entorno y reacciona con él. Entre los hombres y los animales no existen diferencias de principio, sino solamente graduales y de modo. Las orientaciones se orientan con más fuerza en otras orientaciones. Son las más beneficiosas unas para otras, pero también las más peligrosas. Son individuales, tienen ya sus propios puntos de vista, horizontes, perspectivas, puntos de referencia, señales y mundos de orientación. En lugar de iniciar con antropologías generales y doctrinas acerca del hombre en cuanto tal, que aparentan validez general, se debe comenzar con las orientaciones de hombres particulares hacia otros hombres particulares, en las que siempre permanecen márgenes de decisión, también en contra de definiciones generales.

Palabras clave: Idealización/desidealización, normalización/ desnormalización, orientación en el otro, Feuerbach, Nietzsche, Luhmann.

\section{Abstract}

Anthropology suffers from the dilemma that it generally speaks of human beings, whereas we are always dealing with individual human beings. It arises in the course of what Nietzsche calls the 'death of God': When Feuerbach replaces theology with anthropology, he embellishes the image of man, which thus becomes an ideal and a norm. Darwin, Nietzsche, and Freud, and to some extent also the philosophical anthropology of the 20th century, seek to depart 
from this: From the point of view of evolution, the animal human being is an ongoing experiment that overcomes every attempt to define a timeless being over the course of time. One century after Nietzsche, Luhmann deidealizes and de-normalizes 'man': He dissolves the apparent unity of man into three systems, the physical, the psychical and the societal system of the communication, which observe each other and react to each other without determining each other. The leeways that remain in the relationship between the systems, which are each other's environment, can best be grasped by the concept of orientation: Everything that orients itself, whether body, consciousness or society, is an observation system that orients itself in its environment and reacts with it. There are no differences in principle between humans and animals, but only gradual and modal differences. Orientations orient themselves most strongly to other orientations; they are most helpful for each other, but also most threatening. They are individual, always have their own points of view, horizons, perspectives, clues, signs and worlds of orientation. Instead of general anthropologies, seemingly universally valid teachings about human beings in general, one must therefore begin with the orientations of individual people in relation to those of other individual people, in which there is always room for decision, even in relation to general provisions.

Keywords: Idealization/de-idealization, normalization/de-normalization, orientation to other, Feuerbach, Nietzsche, Luhmann. 


\section{Necesidad de la antropología}

Se cae en un dilema cuando se practica antropología, antropología filosófica. Si se entiende 'antropología' en sentido amplio, según el cual se trata de un discurso (lógos) acerca del hombre (ánthropos), toda la filosofía puede ser enmarcada en ella, pues sea como sea, siempre se trata es acerca del hombre $\mathrm{y}$, ante todo, son siempre hombres quienes filosofan, y filosofan a su manera ${ }^{3}$ (Kant, 1962, AA IX, 25). Evidentemente se tiene que contar, aún en las más elevadas generalizaciones filosóficas, con lo humano, demasiado humano, como lo llamó Nietzsche, y por esa razón se distinguen filosofías según nombres propios como, por ejemplo, aristotélicas, kantianas o heideggerianas. Pero si se delimita la antropología filosófica como disciplina de la filosofía, se debe dar forma a un concepto de hombre junto a conceptos de otros objetos de la filosofía tales como 'ser', naturaleza, conocimientos, espíritu, moral, arte, religión, etc. 'El hombre' se vuelve entonces un concepto que se separa de los demás y se tiene que definir de manera universal ${ }^{4}$ (Heidegger, 1929, p. 201). A causa de su subjetividad, se prescinde de los contextos 'del hombre' en su mundo y de los hombres individuales que lo definen y que son definidos a través de él. Lo que queda es una abstracción aislada y, sin embargo, fue visiblemente grande la necesidad de una antropología en los siglos XIX y XX.

Esto se hizo manifiesto cuando Kant fundamentó críticamente toda objetividad en la subjetividad y al mismo tiempo mostró que la existencia de Dios era incognoscible, cuando a finales del siglo XVIII la 'muerte de Dios' estaba a la vuelta de la esquina, y con ésta el 'nihilismo', un concepto ya acuñado por Friedrich Heinrich Jacobi (Müller-Lauter, 1984, pp. 846853). Schleiermacher reaccionó ante esto mediante su teología de 'religiónaun-sin-un-Dios' en el sentimiento de 'total dependencia' del hombre, y de esta manera también puso la filosofía de la religión en un nuevo camino. Rousseau propagó una 'religión civil', una religión de la ciudadanía de los ciudadanos y coronó de esta manera a la fílosofía política. Kant mismo creó,

\footnotetext{
3 "Pero, en el fondo, todo esto se podría incluir en la antropología [las preguntas: ¿Qué puedo saber? ¿Qué debo hacer? ¿Qué puedo esperar? ¿Qué es el hombre?], porque las tres primeras preguntas se remiten a la última".

4 "La antropología filosófica se convierte, entonces, en una ontología regional del hombre, y como tal queda asociada a las otras ontologías, que se reparten con ella el dominio total del hombre". Así pues, para Heidegger ella no es originaria y tampoco es filosóficamente suficiente.
} 
por incitación de Moses Mendelssohn, una filosofía de la orientación (“¿Qué significa: orientarse en el pensamiento?") y satisfizo de este modo una necesidad de la misma filosofía crítica. Todo esto presuponía un concepto de hombre aún determinado por su referencia a Dios, pero que ahora se encontraba en una tensión no resuelta con él. Solo a mitad del siglo XIX Feuerbach resolvió esta tensión con un programa que declaraba hacer nacer de la teología del Dios ahora muerto, una nueva antropología, y hacer su Dios al hombre mismo que hasta entonces había proyectado su mejor cara en un más allá de carácter divino ${ }^{5}$. En Dios, el omnisciente y todopoderoso, habría de conocerse el hombre a sí mismo y de esta manera no avergonzarse más de su corporalidad, degradada durante milenios por las antiguas religiones, sino gozar completamente de ella, y de esta manera estar pleno en el mundo. A partir de esto habría que pensar el resto. La antropología se convirtió en una disciplina fundamental de la filosofía, pues fue al mismo tiempo una disciplina y la filosofía en su conjunto; el dilema parecía disipado.

Sin embargo, desde entonces se multiplicaron constantemente las antropologías filosóficas. Aquello que 'el hombre' es, siguió siendo problemático, y tanto más creció entonces la necesidad de asegurarse por sí mismos de la 'muerte de Dios'. El concepto de hombre que fue necesario crear para dar este paso debió estar, pues, tan saturado de experiencia como fuera posible, y tener en cuenta integralmente a las correspondientes ciencias empíricas. Simultáneamente se continuó buscando al hombre en las huellas de las definiciones esenciales de Aristóteles, según las cuales él debería estar comprendido en algo que autónoma (chooristón), separada e independientemente de otro, no estuviera expuesto a sus influencias y tampoco estuviera sujeto al tiempo. Pero la definición metafísica de la esencia tenía, por su parte, su medida más alta en la esencia del Dios absolutamente autosuficiente. De este modo surgió un nuevo dilema: el hombre, expulsado de la scala naturae aristotélica, del ordenamiento en la escala secuencial de las plantas y los animales hasta Dios, fue concebido instar Dei, a imagen de Dios. Lo que pretendió Feuerbach, la definición esencial del hombre a partir de la esencia del Dios antiguo, se

5 Esto a lo cual alude Odo Marquard en su legendario artículo sobre antropología (1971, pp. 363 y 367), se abrió paso desde el siglo XVI y con ello se invirtió el sentido del concepto de 'antropología': significó ante todo 'hablar del hombre como de Dios'. En tal sentido este concepto fue desplazado por el de 'antropomorfismo" "y -ya sin empleo- quedó libre para un nuevo significado", el significado 'hablar del hombre como de Dios'. Debemos prescindir aquí de la competencia de la antropología con la filosofía de la historia en el siglo XIX, donde Marquard pone el acento.

Stegmaier, W. (2020). La orientación en los hombres. Acerca del problema de la 
hallaba ya en la lógica de la definición de la esencia. Sin embargo, como ella condujo a conceptos esenciales del hombre tan diversos como polémicos, sólo quedó renunciar a ella, como hizo por ejemplo Heidegger, o bien configurar de forma normativa el concepto de esencia, tal como especialmente ocurrió. En consecuencia, el concepto ya no debería corresponder al objeto, sino el objeto al concepto: un concepto normativo es resistente al desengaño, de manera 'contrafáctica' pasa libremente por alto las 'realidades' a las que luego de la transformación de la teología en antropología querría asegurarse en conexión con las ciencias empíricas. Y las éticas no tienen lugar de una manera menos diversa que las antropologías. El dilema de las contradictorias definiciones esenciales del hombre se mantuvo y creció.

Esto ya fue un error de nacimiento de la antropología filosófica. El Dios no sólo concebido, sino también objeto de creencia en la tradición judeocristiana, había ofrecido durante milenios a la orientación humana el más alto sostén en Europa. Sin embargo, en la medida en que él negó expresamente al hombre una imagen y un concepto de sí mismo, permaneció al mismo tiempo imprevisible para ellos, y tal como la teología tenía que hacer a Dios entendible y previsible ${ }^{6}$, al menos hasta un cierto grado, así tenía que hacerlo ahora la antropología con el hombre. La teología había suprimido paulatinamente los rasgos duros y crueles de Dios referidos por la biblia hebrea, dejando sólo un Dios bueno y amoroso. Algo similar sucedió entonces con el hombre. Si para Aristóteles el hombre había sido, por un lado, el ser vivo más noble pero, por otro lado, el más imprevisible y capaz de lo peor (1988, I 2, 1253a32-37), por lo cual debería precisamente tomar medidas en la naturaleza ordenada por Dios, cuando se derrumbó progresivamente en la Modernidad la creencia en un orden de la naturaleza verdadero, bueno y bello, el hombre se volvió, en consecuencia, cada vez más peligroso, y el humanismo tomó en consecuencia su impulso: el gran programa estético-moral de educación hacía del hombre malo uno bueno, del hombre indigno uno digno.

El verdadero concepto de hombre, ahora venido abajo respecto a la altura de Dios, de ningún modo fue afirmado como realidad presente, sino apenas prometido al futuro. Sólo si larga e insistentemente se muestra al hombre la imagen más buena posible de sí mismo, la imagen de un ser que busca la

\footnotetext{
${ }^{6}$ Nota del traductor: (un)berechnbar significa tanto (im)previsible como también (in)calculable. En adelante el término se traduce optativamente según el contexto. Lo mismo vale para Berechenbarkeit: calculabilidad o previsibilidad.
} 
verdad, practicante de justicia y viviendo en amor, él se ajustará con el tiempo cada vez más a ella. La norma limitante se convirtió en ideal prometedor ${ }^{7}$ (Rölli, 2005, p. 358). Precisamente las democracias modernas, en la medida en que renuncian a fundamentaciones religiosas, tienen una profunda necesidad de este tipo de antropología naturalista, cuando no de una religión civil. Si los hombres no tienen ya para 'el hombre' ninguna medida fuera de sí (el bello cosmos) y/o más allá de sí (el buen Dios), deben procurarse ellos mismos una medida, una norma o, mejor aún, un ideal para poder convivir de acuerdo con él. Pero entonces la medida del hombre ya no es su 'naturaleza' -la que originalmente tenía presente la antropología-, sino un orden social. Ella se convierte en antropología política.

Sin embargo, normas e ideales siempre hacen recordar cuánto se desvían de ellos los hombres, qué tan mal y feo se pueden comportar éstos, comparados con sus normas e ideales. Ellos tornan mucho más interesante lo malo y lo feo. Desde la distancia segura de la propia medida ideal, esto ya había sido explorado desde hace mucho tiempo en pueblos considerados 'exóticos' y 'primitivos'. Pero cuando después de Feuerbach se tornaron viables también las famosas 'afrentas' de Darwin, Nietzsche y Freud a los hombres, apareció la antropología humanista haciendo metafísica, de modo tan dudoso que el concepto de 'antropología' fue apropiado cada vez más por ciencias empíricas. La "antropología filosófica” del siglo XX, asociada en particular a los nombres de Scheler, Plessner y Gehlen, se quedó en un estadio intermedio $^{8}$ (Fischer, 2008, p. 516). Hacia finales del siglo XX, después de que lo más malo de la humanidad aflorara de la peor manera, lo 'racional' en la definición aristotélica del 'animal rationale' (zóon lógon échon) fue cada vez menos comprendido en el sentido de que el hombre tenga razón, ya sea de Dios o de la naturaleza, y que por ello siempre sea calculable para todos los demás, pues evidentemente no lo es. En cambio, cada uno en su situación

\footnotetext{
7 Indica que Kant comete "una 'falacia naturalista' en sentido inverso: no concluye sobre el deber a partir del ser, sino sobre el ser a partir del deber"

8 Acerca del intento de "restaurar las intuiciones correctas de la filosofía de la razón en el medio de los descubrimientos correctos de la crítica de la razón" en Scheler, Plessner, Gehlen, Rothacker y Portmann, cfr. Joachim Fischer (2008, p. 516). Él distingue la "antropología filosófica" (escrita con minúsculas) en calidad de disciplina filosófica, de la "Antropología filosófica", como nueva "manera de pensar" (Denkansatz), es decir, como nueva filosofía fundamental (p. 488). Nosotros asumimos tal distinción. Nietzsche, cuyo diagnóstico -"Dios ha muerto"- rápidamente se convirtió en eslogan y pareció hacer que una Antropología filosófica fuera casi obligatoria para la reorientación, permanece completamente al margen junto a Fischer.
} 
particular debe mirar cómo puede, del modo más expedito, contar con los otros con los que tiene que tratar, cómo puede hacer la relación con ellos tan calculable como sea posible 9 (Hoffmann, 1992, pp. 52-66). Bajo la condición básica de su imprevisibilidad, los hombres buscan, o crean, una y otra vez, márgenes de maniobra para su previsibilidad. Lo que se intenta a través de la antropología filosófica es asegurarse mutuamente, mediante idealización contrafáctica, un ser común. En el trato mutuo cotidiano tales definiciones esenciales ayudan poco y pueden, por el contrario, perjudicarlo, como en el caso de que la otra persona no las comparta. Estas definiciones provocan, entonces, confrontaciones ideológicas y de esta manera la antropología filosófica se vuelve contraproducente.

Entretanto, las democracias modernas, evidentemente, se han afianzado tanto que uno se puede permitir ver la parte imprevisible 'del hombre', considerada peligrosa, sin desacreditarla de entrada como irracional, mala o malvada (“... no merece ser un hombre ...”). Los precursores más enérgicos de este 'poder-ver-también-el-otro-lado' fueron Friedrich Nietzsche, a finales del siglo XIX, y Niklas Luhmann al final del siglo XX. Ellos insistieron en liberar del bien y el mal el discurso acerca del 'hombre'. Nietzsche empuñó el 'poder-ver-también-el-otro-lado' bajo el lema 'perspectivismo'; Luhmann bajo el lema 'observación de segundo orden'. En lugar de preguntar por esencias permanentes de los objetos y buscar su definición universal, en una perspectiva aparentemente sin alternativa o una observación de primer orden, en las cuales los objetos aparecen como objetos en sí, aislados de sus contextos, ellos observaron: por quién, cuándo, por qué tales preguntas fueron planteadas y con qué diferenciaciones de por medio se hicieron, para ver qué alternativas aparecen -en este caso, otras posibilidades del 'ser humano'. Ellos querían (independientemente uno del otro, Luhmann apenas se refería a Nietzsche) desmontar el aislamiento metafísico y la idealización moral 'del hombre' y, en cambio, con la clara conciencia de los dilemas mencionados, averiguar qué función o funciones cumple realmente el concepto o el término 'hombre' en la comunicación de la sociedad. Me adhiero inicialmente a Nietzsche y Luhmann, pero sin permanecer posteriormente tras sus huellas. Por el contrario, abordaré en un tercer momento la pregunta por la 'orientación

\footnotetext{
9 Ya para Max Weber 'racionalidad' recibió, además del sentido de racionalidad, el de calculabilidad, "ponderación de medios", "planeación metódica", "ejecución eficiente", "explicación suficiente bajo circunstancias determinadas", que pueden -aunque no deben- culminar en "transparencia lógica" y "justificación plenamente razonable".
} 
en el hombre' a partir de la filosofía de la orientación y responderé con la 'orientación en los hombres', hombres en plural $^{10}$ (Stegmaier, 2008). Al mismo tiempo la necesidad de la antropología se vuelve así explicable y reductible. Teniendo en cuenta la extensión de este trabajo, sólo será posible desarrollar aquí un esquema aproximado a este asunto.

\section{Desidealización y desnormalización del hombre en Nietzsche y Luhmann}

Nietzsche se hizo famoso también por su ruptura declarada con el aislamiento y la idealización de una esencia humana. En su obra habla siempre del 'hombre', lo 'humano' y lo 'demasiado humano', frecuentemente de lo 'antropomorfo' y de 'antropomorfismos'; sin embargo, excepcionalmente de lo 'antropológico' y los 'antropólogos'11 (Nietzsche, Nachlass 1872/73, 19[91], KSA 7, p. 449), y absolutamente nunca de 'antropología'. De modo evidentemente consciente, Nietzsche no escribió algo así como una antropología ${ }^{12}$. Con toda la estimación de Feuerbach, no participó de su transformación de la teología en antropología y renunció, en general, a doctrinas en apariencia universales (Stegmaier, 1995, pp. 214-239; Gerhardt, 2000, pp. 191-224), a una doctrina acerca del hombre. En su lugar, desde su primera aparición entre los hombres en la plaza, Nietzsche hizo polemizar a su figura de Zaratustra contra los

10 "Luhmann encuentra a Nietzsche. Orientación en el nihilismo".

11 "Toda ciencia natural es sólo un intento de entender al hombre, lo antropológico: más exactamente aún, por vía de los más monstruosos rodeos, regresar siempre a lo humano. El abotargamiento del hombre hacia el macrocosmos para al fin decir 'tú eres al fin, lo que tú eres"”, y Götzen Dämmerung ("Das Problem des Sokrates", § 3): "Los antropólogos, entre los criminalistas, nos dicen que el criminal típico es: monstrum in fronte, monstrum in animo").

${ }^{12}$ No obstante, Heinz Heimsoeth esboza una "antropología de Friedrich Nietzsche" (1943), en el apogeo de la antropología filosófica y, a la vez, en la época del nacionalsocialismo. Aunque sin remitirse a ellos, sino solamente concentrado en textos de Nietzsche, Matthias Schlossberger (1998, pp. 147167) señala a distancia histórica en qué lugar Scheler, Plessner y Gehlen están próximos a Nietzsche y dónde siguen estrategias propias. Mientras la antropología filosófica vuelve a preguntar por la esencia del 'hombre', lo que elevaría a éste sobre 'el animal', Nietzsche renuncia tanto como sea posible a tal definición esencial y, en consecuencia, también a una antropología. Geisenhanslücke (1999, pp. 125-140) corrobora esto mediante la fábula del inicio de Sobre verdad y mentira en sentido extramoral y Consideraciones intempestivas $I$, en las cuales Nietzsche elude de entrada toda antropología que insista en un determinado concepto de hombre. Mas recientemente Schacht (2006, pp. 115-132) debe, sin embargo, ganar a Nietzsche para la antropología, en tanto revisa cronológicamente su obra en busca de enunciados antropológicamente relevantes en ella. Él sigue la tesis según la cual la antropología de Nietzsche sería estrictamente 'naturalista', presuponiendo la 'naturaleza' como dada, y sólo debería abrirse sin prejuicio y metódicamente (Schacht, 2005, pp. 277-294). Schacht sostiene entonces la tesis según la cual la antropología de Nietzsche sería "conceptualmente más aguda, metodológicamente más concluyente, científicamente más exigente" que la de Kant. A través de ella la antropología filosófica se vuelve "intelectualmente respetable" (p. 285).

Stegmaier, W. (2020). La orientación en los hombres. Acerca del problema de la 
‘últimos hombres' (Nietzsche, 1988, KSA 4, § 5)'13; Meckel, 1980, pp. 174-208; Gillespie, 1999, pp. 141-155). El 'último hombre' es un hombre interesado en una definición última de la esencia del hombre, válida igualmente para todos y para todo tiempo. Él hace esta definición a la medida de sí mismo, se toma a sí mismo como escala, con la necesidad de sacar de ello el mejor partido. La evolución 'del hombre' debe haber llegado en él -en su mediocridad- a su término. Por el contrario, Nietzsche planteó su famosa 'anti-definición': "hombre" sería "el animal aún no fijado" (2018, § 62; Gehlen, 19714). Nietzsche consideró a los humanos como unos animales entre otros y los ordenó en la evolución junto al resto: la conciencia, la condición de razón e inteligencia, promesa de la más alta humanidad, se vuelve entonces la adquisición más tardía y, comparativamente, aún muy inestable (Nietzsche, 1882, § 11). Ella podría resultar en el futuro tan peligrosa para la vida como favorecedora para ella, tan amenazante para la moral como fomentadora de ella. Su otra cara, la unión de los instintos, tan poco valorada frente a ella, podría resultar, en cambio, conservadora de la vida, mientras el mal -la contracara del bien que se atribuye a él-, podría ser acrecentador de la vida (Constâncio und Mayer, 2011). De todos modos, así habrían crecido vigorosamente culturas antiguas como la griega y la romana. Lo cobijado con el término 'hombre' sería tan abismal como insondable en sus complejas conexiones, mucho más que el

\footnotetext{
${ }^{13}$ Para la exposición de la crítica nietzscheana a la antropología productora de metafísica me limito a los apartes correspondientes al "Prefacio" a Also sprach Zarathustra. Meckel (1980, pp. 174-208) incluye todo Also sprach Zarathustra, mientras Gillespie (1999, pp. 141-155) el discurso "De las tres transformaciones", con el cual comienza la parte I. No me puedo adherir a su asociación de (la crítica a) la antropología y el nihilismo. Según las interpretaciones fundamentales de Meckel la "antropología de Nietzsche" gira en torno al hombre, que en la creación, el amor y la lucha contra el espíritu de la pesadez, va más allá de sí.

${ }^{14}$ Gehlen ha destacado el equívoco en la fórmula: "Eso quiere decir, primero, que no hay aún una constatación de aquello que realmente sea el hombre y, segundo, que la esencia humana es, de algún modo, inacabada, no está 'establecida con firmeza". Porque la 'esencia' del hombre sólo puede consistir en su definición, ambos son inseparables. Tal como Michel Foucault lo mostró luego detalladamente, la 'esencia humana' misma es una "invención reciente" del discurso filosófico y científico de los siglos XIX y XX, a la cual uno se aferró en vista de la "muerte de Dios", y de la cual también se desprenderá de nuevo ("fin del hombre") cuando esta última se haya asimilado, preparada ahora por la propia "arqueología" foucaultiana de la evolución del "orden" de estos discursos (Foucault, 2003, pp. 460-462). Acerca del contacto del primer Foucault con Darwin y Nietzsche cfr. Erb (2010, pp. 137-148). La 'arqueología' de Foucault siguió igualmente, a su manera, el método del 'poder-ver-también-el-otro-lado'. De esta manera se habría desarrollado el pathos de la razón en el rechazo de la locura que amenaza permanentemente (Foucault, 1961). Junto a Nietzsche, para quien esto era claro, resultó de ello luego, según Erb (2010, p. 145) "un (...) tipo de "contra-antropología (...) dionisiaca". No es posible proseguir aquí con la conexión de Foucault y Nietzsche en lo que respecta a la (contra-) antropología.
} 
cuerpo, ya demasiado complejo, y que podría servir de momento, al menos, como hilo conductor del acceso a esta complejidad (Schipperges, 1975; Lemm, 2009; Figl, 1982, pp. 124-139). Nietzsche siguió explícitamente el programa de una desidealización y una desnormalización, de una "naturalización" del hombre; programa de disolución de la antropología aún próxima a la metafísica, mediante una tipología histórica y cultural de la diversidad de los hombres ${ }^{15}$, y con ello también fue más allá de la lógica aristotélica de la definición: "definible es sólo Eso que no tiene historia" (Nietzsche, 1887, II $\S 13)$. En tanto determinaciones últimas, las definiciones prescinden de la evolución o la tratan como concluida.

Desde el punto de vista de la evolución el animal humano es, por el contrario, un experimento constante. Quien lo sepa puede entender también su propia humanidad de esta manera y, si es filósofo, con una observación de segundo orden buscará o creará diferenciaciones que permitan entender esto del modo correspondiente. Él pasará de unidad a diferencia y de esencialidad a temporalidad y esperará, bajo las respectivas nuevas circunstancias, la necesidad de un nuevo 'ir-más-allá-de-sí', ya no en el sentido de un progreso hacia un fin, sino sólo en el sentido de la apertura de otros márgenes de maniobra para ulteriores despliegues. Para eso necesitó Nietzsche la fórmula del superhombre ${ }^{16}$ (Visser, 1999, pp. 100-124).

Con esta fórmula Nietzsche ofreció tal doctrina -superficialmente, 'exotéricamente', como él la llamó (Nietzsche, 2018) - a quienes esperan y necesitan una doctrina general del hombre, para mostrársela 'esotéricamente' como insostenible a quienes han aprendido a leerlo más detalladamente y

${ }^{15}$ Cfr. Nietzsche (1882, § 109) y Nietzsche $(2018, \S 230)$, y más frecuentemente y con ese fin, Bertino, especialmente el pasaje 2.5.2: Nietzsche: "Die Überwindung der Anthropologie zugunsten der Typologie" (2011, pp. 207-210).

${ }^{16}$ A partir de la fórmula 'superhombre' Visser pregunta nuevamente por el sentido del 'hombre' en ella, con el resultado (desde mi punto de vista, correcto), de "que para Nietzsche, en el fondo, no se trataría del hombre" (1999, p. 103). Él remite para ello a los Nachgelassene Fragmente (1883/1884, 24[16], KSA 10, pp. 655s.): "al fin y al cabo no se trata del hombre: él debe ser superado". Por el contrario, no puedo seguir la tesis de Visser según la cual la separación de la antropología que hace metafísica llevaría al criterio exclusivo de lo orgánico y con él al racismo (p. 105), tampoco sus otras declaraciones sobre "los secretos del ser-humano" (p. 117). Cfr. finalmente, para la discusión sostenida aún con intensidad acerca del sentido de la figura del "superhombre" de Nietzsche, en contraposición con el "último hombre" (Skowron, 2013, pp. 256-282, especialmente 270-273), quien también añade, entre las divergencias, los tipos nietzscheanos de los hombres 'ordinarios', 'reales', por una parte, y 'superiores', por otra. Sobre el sentido múltiple del 'super-' en 'superhombre' cfr. pp. 274-277. 
se han liberado de la necesidad de conceptos metafísicos esencialistas ${ }^{17}$, pues el superhombre, por su parte, no es un tipo, un género con su esencia común, sino siempre un individuo, y la evolución, que se puede contemplar en retrospectiva como "acrecentamiento de la especie "humana" (Nietzsche, 2018, § 44) o "del tipo 'humano" (§ 257), es impulsada siempre por individuos, por individuos que bien pueden cooperar de muy diversa manera, pero que no están obligados a colaborar con ello (Nietzsche, 2016, § 4). Es por esto que Nietzsche no asoció con la fórmula 'superhombre' ningún darwinismo social, ningún programa para la imposición de los 'más fuertes', sea un estamento, una clase o una raza. Para él fue claro que, en primer lugar, se debía señalar en cada caso qué, cuándo y dónde se constituye 'fuerza' en la evolución (Stegmaier, 1987, pp. 264-287). Un 'superhombre' únicamente es, entonces, un hombre que va más allá de las posibilidades actuales del resto de los hombres y es superior a ellos, por ejemplo, como descubridor, artista, científico, político, transmutador de valores o fundador de una religión. Sin embargo, en otros aspectos puede ser inferior y, por ello, también hundirse fácilmente bajo ellos, como lo muestra Nietzsche en el Zaratustra cuando permite que éste se marche con los ‘últimos hombres'. Especialmente cuando se trata de moral, de transmutación de valores, parecerá malvado según los valores vigentes $\mathrm{y}$, en consecuencia, estará en mayor peligro que los otros.

La fórmula 'superhombre' actúa así, con su particular "luminosidad", como "contraconcepto ${ }^{18 "}$ (Nachlass 1888, 23[3], KSA 13, p. 603) frente a todo concepto fijo y último 'del hombre'. En tal sentido, ni siquiera es un concepto: Nietzsche no lo introdujo en conceptos definitorios (lo que a menudo es deplorado), sino en una red de metáforas que siempre mantiene abiertos nuevos desarrollos conceptuales (Stegmaier, 2010, pp. 145-179). Nietzsche rechazó una antropología del autoaseguramiento que se hace mediante la

\footnotetext{
${ }^{17}$ Así como él, exotéricamente, propuso con la fórmula 'eterno retorno de lo mismo' toda una metafísica (no existe, expresamente, 'lo mismo' según Nietzsche, y como 'eterno' deberían valer justamente las esencias metafísicas), para volverla paradójica esotéricamente mediante la introducción del tiempo en la eternidad ('retorno'). Algo similar vale para la fórmula 'voluntad de poder'. Las tres fórmulas están dirigidas a la destrucción de conceptos metafísicos esenciales -siempre para lectoras y lectores necesitados de metafísica. Nietzsche beatifica expresamente con ellos a sus lectoras y lectores. Ellos debieron 'comprometerse' con una nueva comprensión metafísica de su destrucción de la metafísica. Esto le ocurrió al mismo Martin Heidegger. Cfr. Werner Stegmaier (2011b, pp. 160-170).

${ }^{18}$ Así es como se tiene que entender también su fuerte sobrevaloración retórica, detrás de la cual no se debe perder de vista su sentido objetivamente factible.
} 
inmovilización conjunta de todo bajo un concepto $^{19}$ (Nachlass 1880, 6[158], KSA 9, p. 237). En lugar de eso, con su 'psicología' y su 'genealogía' exploró críticamente el trasfondo de las determinaciones precedentes para negar su aparente certeza. Asoció, en suma, su anti-antropología con su planteamiento en torno a la certeza:

(...) nosotros tomamos una posición distinta frente a la 'certeza'. Porque durante mucho tiempo el miedo ha sido cultivado en el hombre y toda existencia soportable comenzó con el 'sentimiento de seguridad', así continúa obrando esto todavía ahora en los pensadores. Pero tan pronto la 'peligrosidad' exterior de la existencia retrocede, surge un placer por la inseguridad, inmensidad de la línea de horizonte. La fortuna de los grandes descubridores en el afán de certeza podría transformarse ahora en la fortuna de demostrar que hay por doquier incertidumbre y riesgo (Nachlass 1884, 26[280], KSA 11, pp. 223s.).

Cien años después el sociólogo Niklas Luhmann prosiguió con la pregunta por 'el hombre' en esa misma dirección, precisamente con la objetividad moral y filosófico-moral por la cual Nietzsche siempre luchó y que ahora encontró una mayor resistencia por parte de antropologías humanistas aún más afirmadas. La frescura, incluso frialdad, de sus observaciones y concepciones teóricas es para la mayoría aún más difícil de soportar que la 'ciencia jovial' de Nietzsche. Luhmann igualmente había estudiado con intensidad la filosofía y la incorporó con maestría en su sociología: pues aquella es también parte de la comunicación de la sociedad y ha contribuido por milenios a su semántica. Desde este punto de vista también Luhmann preguntó por 'el hombre': cómo sería éste como partícipe en la comunicación de la sociedad y cómo estaría determinado por ella. Incluso desidealizó y desnormalizó 'al hombre' de este modo e insistió, a distancia de todo lo 'contrafáctico', en la observabilidad estricta -y pudo entonces echar mano de otros cien años de investigaciones antropológicas empíricas. Dejó atrás,

\footnotetext{
19 "Tan pronto como queramos determinar la finalidad del hombre, anteponemos un concepto de hombre, aunque sólo hay individuos. A partir de lo hasta ahora conocido, el concepto sólo se puede obtener excluyendo lo individual - es decir, establecer la finalidad del hombre significaría imposibilitar a los individuos en su devenir individual y llamarlos devenir común. ¿No debería ser cada individuo, a la inversa, el intento de alcanzar un género más alto que el de los humanos mediante sus cosas más individuales? Mi moral sería la de tomar del hombre cada vez más su carácter general y especializarlo, hasta el punto de hacerlo más inentendible para los otros (y así objeto de experiencias, de asombro, de instrucción para ellos)". Visser (1999, p. 107), sostiene correctamente: "Así pues, el fin del hombre para Nietzsche es el fin del concepto "hombre"”.
} 
como Nietzsche, la vieja intención ontológica de querer determinar en conceptos universales lo aparentemente fijo en sí y siguió decididamente la opción teórica de la diferencia: de una u otra manera diferenciar lo que de una u otra manera es diferenciable, observar los conceptos como partes de diferenciaciones que siempre tienen otro lado, uno alternativo. Las diferenciaciones no son entonces reproducciones de lo comprendido, sino decisiones de quien comprende. Entendido de esta manera, todo comprender que deba crear certeza engendra a la vez incertidumbre, porque siempre sería posible decidirse también por el otro lado de la diferenciación. No obstante, dicha incertidumbre puede ser siempre el impulso para una comprensión más amplia. De este modo, también el concepto 'hombre' permanece abierto a sorprendentes evoluciones: "la fórmula 'hombre' [es] solamente aún un concepto de unidad o concepto marco para una complejidad manifiesta, pero ya no un objeto sobre el que se puedan formular enunciados directamente" 20 (Luhmann, 1995b, p. 269).

Diferenciaciones son operaciones de observaciones y las observaciones, por su parte, presuponen la diferenciación entre un observador y un observado. Luhmann concibió esta última en su teoría de sistemas como diferenciación entre sistema y entorno. Los 'sistemas' son sistemas de observación que, para poder observar su entorno, para estar 'abierto' a él, deben diferenciarse de él, delimitarse, cerrarse y, en este sentido, al mismo tiempo estar 'clausurados'.

Por esta razón el entorno observado es siempre más complejo que el sistema, que debe escoger y de esta manera reducir la complejidad ${ }^{21}$. Para el sistema, en sus observaciones, se trata entonces siempre de una complejidad reducida,

\footnotetext{
${ }^{20}$ En gran medida Luhmann es un pionero en la sociología, como Nietzsche lo fue anteriormente en la filosofía. La semántica humanista se afirma también aquí empecinadamente, e incluso resulta difícil a los sociólogos "desistir del hombre" (Luhmann, 1995b, p. 273). Sobre la función de la semántica humanista de "orientar la desorientación" en un "período de tránsito" (desde una diferenciación social estratificadora a una funcional), y transformar las "condiciones de posibilidad" (en tanto desteologiza cuidadosamente lazos religiosos duraderos), cfr. Niklas Luhmann (1980, pp. 170 y 173). Uwe Schimank (2005, pp. 265-284) proporciona una revisión crítica de la crítica de Luhmann a la teoría - particularmente humanista- del hombre desde una perspectiva sociológica. Sin embargo, su declarado "individualismo metodológico" (p. 275) lleva a crasas distorsiones, según las cuales Luhmann se limitaría sólo a la coordinación y pasaría por alto el conflicto (p. 269), y las personas individuales serían para él "socialmente insignificantes e intercambiables" (p. 271). Al final lo atribuye a la juventud de Luhmann en la era nazi (pp. 281s.).

${ }^{21}$ En esto se puede ver, según Schimank (2005, p. 268), un enunciado antropológico, una "antropología mínima”. Sí, sólo que no una antropología ‘humanista' apoyada en la razón.
} 
nunca puede asirla completamente y observar (en observaciones de segundo orden) que otros sistemas de observación observan de otra manera, no sólo en la vida cotidiana, sino también en la ciencia. En cada sistema de observación es todo diferente, pero esto no es lo que debe ser explicado, sino el hecho de que la improbable creencia en la igualdad se vuelva, sin embargo, factible y se acredite, y cómo sucede eso. Con ello se alcanza el perspectivismo de Nietzsche.

Según la vieja intención ontológica con la cual estamos aún habituados a pensar, 'el hombre' es el elemento de la sociedad, aquello de lo que ésta se construye. Es por ello que la antropología humanista, que hace metafísica, piensa al hombre idealmente conforme a la sociedad (Luhmann, 1984, p. 286). Aunque en realidad todo lo que en el hombre es llamado tradicionalmente 'cuerpo' y 'alma' -y que todavía Nietzsche denominó así-, los complejos y complejamente entrelazados sistemas fisiológicos que posibilitan su vida biológica, no es parte de la vida social, tanto menos lo es aquello que desde William James y Edmund Husserl es denominado 'flujo de conciencia', el decurso de representaciones inicialmente desordenado. No obstante, ambos son, por su parte, sistemas de observación autónomos y observan cosas diferentes: el cuerpo, que por esta razón Luhmann llamó 'sistema físico', es lo relevante para el desarrollo de la vida corporal (por ejemplo, la temperatura exterior fluctuante para mantener la temperatura corporal o el suministro de alimentos para la homeostasis de las células, los órganos de la circulación o los peligros exteriores mediante la activación de los reflejos), mientras el alma, la conciencia o el 'sistema psíquico', es lo perceptible del entorno del cuerpo. Sus procesos fisiológicos permanecen principalmente inconscientes; la consciencia reacciona a ellos sólo eventualmente, en sensaciones como dolor o placer, pues con todo lo demás irremediablemente se sobrecargaría, se desbordaría y se desdibujaría el flujo de consciencia ${ }^{22}$ (Gehlen, 1971, p. 70; Loukidelis, 2012, pp. 117-127). En lugar de eso, los procesos fisiológicos, en particular cerebrales, provocan percepciones a través de las cuales son aislados (tal como Luhmann siempre recalca, el cerebro mismo no siente) 'objetos' del entorno, entre ellos también hombres y señales de sus consciencias. Las estructuras de orden de tales señales, en las que estas últimas logran tener

\footnotetext{
22 "De la increíble complejidad y perfección de las ejecuciones mismas, vegetativas y motoras, no tenemos conocimiento y la consciencia, evidentemente, no está allí para instruirnos al respecto". Loukidelis (2012, pp. 117-127) señala nuevamente la cercanía de Gehlen a Nietzsche (no sólo) en este punto. Una "antropología en Nietzsche" está allí presupuesta, mas no expuesta.
} 
sentido para diferentes sistemas psíquicos, de modo que nuevamente puedan ser respondidas por señales con sentido, presuponen a los sistemas psíquicos en un tercer tipo de sistema: el sistema de comunicación de la sociedad con sus múltiples subsistemas. Dichos procesos solo 'hacen partícipes' a estos sistemas, solo desencadenan su operar y lo mantienen en marcha. Se actúa por motivos físicos y psíquicos, y se justifica la acción en caso de que sea necesario, por razones que el sistema social ofrece y admite, pues el sistema físico y el psíquico no tienen razones. Por ello los sistemas de comunicación deben ser considerados autónomos también ante los comunicadores, que deben ser vistos por esa razón como entorno de dichos sistemas. Por su parte, los comunicadores se diferencian según inclusión o exclusión de la comunicación, conexión en la comunicación o expulsión de ella, y también observan en dirección a tales diferenciaciones. Independientemente de la conciencia individual, aquellos determinan cómo se debe hablar y qué se puede decir, cómo se tiene que actuar y cómo no hacerlo, qué roles o funciones tomar, qué se puede reivindicar, gozar o esperar y qué no, qué se tiene que compartir y justificar, etc. Así como el sistema físico (se puede respirar más rápido arbitrariamente, pero no digerir más rápido), también el psíquico puede influir en lo social sólo de manera puntual (aquí o allí se puede separar de sus reglas y también contribuir a sus transformaciones, pero no se puede luchar contra todo el sistema o ignorarlo), y, por su parte, también el sistema social frente al sistema psíquico y al sistema físico. Los sistemas, mutuamente autónomos, siguen jugando su propio juego en su interacción, lo que ya había motivado a Nietzsche a ponerlos a jugar unos contra otros.

De esta manera la teoría de sistemas ha logrado en medio de estos (sistemas) la libertad de maniobra que (aquí) se puede apreciar. Los hombres pasan de ser partes de la sociedad a ser el entorno de la sociedad, la que a su vez está compuesta exclusivamente de comunicación. Ella 'existe' sólo porque comunica y, como sociedad, sólo porque se comunica (algo) acerca de ella, según diferenciaciones no de individuos, sino nuevamente de la misma sociedad. Como hombres, o más exactamente como sistemas psíquicos o conciencias, 'toman parte' en la sociedad. Cómo se tiene que explicar que la comunicación se dirige a ellos y como parte de ellos, son cuestiones que deben quedar de momento abiertas, así como el proceso que posibilita que la conciencia genere el comportamiento corporal (que la mano se levante cuando ella 'quiere') (Luhmann, 1995a, pp. 37-54). Sin embargo, se puede observar que la comunicación "fascina y ocupa" la conciencia mediante el lenguaje y la escritura en los que ella se lleva a cabo. Esto sucede de 
manera manifiesta porque lo mismo ocurre con todas las cosas perceptibles, con incomparables objetos de la percepción (pp. 40ss.). La conciencia es "penetrada", según Luhmann, por la comunicación; incorpora las estructuras de esta última en las propias, pone a disposición de ella un "espacio libre" en el que el sistema social puede operar y construir su propia complejidad ( $\mathrm{p}$. 41). La comunicación emplea estos espacios libres, lo que entonces se puede concebir teóricamente como "medio" fluido (¡flujo de conciencia!), en el cual ella configura "formas" fijas que, por su parte, saltan a la vista como tales y son recordadas en consecuencia (p. 44), mientras, por lo demás, el flujo de conciencia cae rápidamente en el olvido. Es por ello que un individuo (un sistema psíquico individual) sólo puede decir aquello que puede decir sobre sí mismo en estructuras del sistema social de comunicación, y no precisamente de modo individual, tal como Nietzsche ${ }^{23}$ señaló $(1882, \S 354)$. Cuando un individuo habla, habla también acerca de sí para sí mismo, habla ya dentro de los ordenamientos del sistema social. Pero él no solamente está "fascinado y ocupado" por el sistema, sino que tiene también la "posición privilegiada para poder interferir, estimular, irritar la comunicación", empezando con estimular la comunicación de sus percepciones -para las que, a su vez, es estimulado por el sistema físico- que permanecen siempre como sus percepciones singulares. Los mismos sistemas de comunicación tampoco pueden percibir, ya que sólo procesan "campos de percepción” de sistemas psíquicos. En este sentido, los sistemas de conciencia y de comunicación mutuamente implicados, aunque autónomos unos de otros, desencadenan operaciones mutuamente respectivas, sin determinarlas, sin embargo (Luhmann, 1995a, p. 45) - precisamente por eso deben ser tratados como sistemas distintos y la unidad 'del hombre' debe ser disuelta ${ }^{24}$.

De lo anterior puede sostenerse, primero, que un sistema psíquico está ligado a uno físico, porque los procesos psíquicos no transcurren sin procesos cerebrales, ni los procesos cerebrales sin los procesos fisiológicos restantes. El yo está "siempre allí donde su cuerpo está, y desde allí puede ocuparse de todas las cosas posibles sin perderse en el mundo exterior a sí mismo. Él sabe siempre dónde está y en qué lugar centra su percepción y su pensar" (Luhmann, 2011, pp. 422s.). En segundo lugar, se plantea que un

\footnotetext{
${ }^{23} \mathrm{O}$ incluso ya Friedrich Schiller: “¿Por qué no puede el espíritu viviente aparecer al espíritu? / Habla el alma, así habla, ay! Ya no más el alma" (Schiller, 1797, p. 177).

${ }^{24}$ Sobre las objeciones desde la perspectiva tradicional de la scala naturae aristotélica como la expone, por ejemplo, Ralf Dziewas (1992, pp. 113-132), cfr. la postura de Luhmann en Dziewas (p. 385).
} 
sistema social sólo se puede formar y operar gracias a hombres concretos y, tercero, que psíquica y físicamente los hombres concretos “[pueden] ser tan complejos como son (Luhmann, 1984, p. 304) solo gracias al sistema social de la sociedad (...)". Sin embargo, ya no es necesario considerar la 'definición del hombre' como ser o devenir un miembro bueno y noble de una sociedad buena y noble, ni tampoco asumir esto bueno y noble como predeterminado por la naturaleza o prescrito por la razón. Luhmann distinguió al 'hombre concreto' (como sistema físico y psíquico) de la 'persona' en la comunicación de la sociedad; 'persona' entendida como un "símbolo de la capacidad de participar en la comunicación", como "marca de identidad" social.

Por inclusión en la comunicación se inculca "calculabilidad" a la persona y en adelante le es supuesta esta "calculabilidad", pero por lo demás, el hombre sigue siendo para la comunicación (y con ello también en gran parte para él mismo) una "caja negra" en el entorno del sistema social (Luhmann, 2011, pp. 90-92). Sin embargo, ya que el entorno es "en comparación con el sistema, precisamente, aquel campo de diferenciación que presenta mayor complejidad y menor ordenamiento", por parte de la teoría de sistemas se conceden a esta caja negra que es el hombre "mayores libertades en relación con su entorno, en particular libertades de comportamiento irrazonable e inmoral" (Luhmann, 1984, p. 289), que él, en fin, tiene como observables, aun cuando no se le quieran reconocer (y eventualmente aun cuando él tampoco las quiera reconocer). Como unidad "guiada por diferencias" (p. 328), de diferentes sistemas autónomos, el hombre sigue siendo sorprendente para el sistema social, así como para cualquier otro sistema psíquico; un experimento incesante, en el sentido de Nietzsche. Aún para Luhmann, los individuos no se insertan fácilmente en el orden social, sino que por el contrario "ellos aportan su vivacidad, por así decirlo (...) su contribución es precisamente la inestabilidad, la que posibilita construir otro sistema, es decir, un sistema social mediante procesos de selección (Luhmann, 2008, p. 84). En lugar del contraconcepto del superhombre, Luhmann pone a salvo el espacio de la libertad humana a través de la teoría de sistemas.

\section{Orientación en los hombres}

Como teórico declarado, Luhmann observa desde un punto de vista teórico para poder encontrar y discutir en torno a él, incondicionalmente, decisiones teóricas, libre de condiciones 'humanas, demasiado humanas'. Nietzsche cuestionó siempre la posibilidad de un punto de vista teórico semejante, en el que uno creería sustraerse de los contextos de la propia vida y comprender 
'desinteresadamente' sus estructuras generales de modo universal, pues de hecho si esto fuera posible, uno saldría por completo de la vida para abandonarse, como los metafísicos lo habrían hecho durante bastante tiempo, a una visión de conjunto divina, desde un más allá ficticio de construcciones ilusorias. Nietzsche mismo, de modo consecuente, no desarrolló un 'sistema' en el sentido de una teoría que encontrara su justificación en su propia coherencia $(1889, \S 26)$, sino que exploró en contextos 'vivientes', en el incesante cambio de perspectivas de sus aforismos, tales contextos. Con ello hizo posible experimentar su propia perspectiva humana, demasiado humana; entró en relación con sus lectores y lectoras mediante un trato personal ('¡oh, amigos míos!') y siempre les dirigió la palabra desde las propias perspectivas de ellos. De la misma manera, en sus abstractas tematizaciones filosóficas, puso visiblemente en perspectiva 'a los hombres' a través de los hombres ${ }^{25}$. Luhmann conocía, por supuesto, los problemas del punto de vista teórico $\mathrm{y}$, sin embargo, también reflexionó en torno a ellos desde su punto de vista teórico, con el argumento convincente de que la condición de la ciencia sería, pues, prescindir de las propias condiciones de vida humana y de las circunstancias personales, toda vez que estas deberán ser comprendidas en general y reconstruidas teóricamente, y de esa manera deben ser abiertas alternativas funcionales para ellas.

Las correspondientes perspectivas humanas interesan aquí sólo en cuanto pueden menoscabar la teoría. Incluso Nietzsche puede evitar seguir hablando de modo abstracto acerca 'del hombre'. Así, en el caso 'del hombre', la teoría como tal plantea un dilema que, precisamente porque ella misma está implicada, ya no puede resolverse a través de ella. Sin embargo, el dilema se disipa si se retrocede hasta el concepto que desde Moses Mendelssohn e Immanuel Kant pasó de geográfico a filosófico y, desde entonces, atraviesa todos los debates filosóficos, científicos y cotidianos (y también los escritos de Luhmann), sin que generalmente sea reflexionado expresamente: el concepto de orientación ${ }^{26}$. La orientación que precede a toda teoría, puede ser descrita

\footnotetext{
${ }^{25}$ Por eso uno debe también atenerse, finalmente, a la obra de Nietzsche publicada o destinada a publicarse, no a las notas póstumas, en cuya versión preliminar aun prescinde, la mayoría de las veces, de esta puesta en perspectiva.

${ }^{26}$ Luhmann rechaza la objeción según la cual "al fin y al cabo serían pues siempre hombres, individuos, sujetos, los que actúan o bien comunican" (Luhmann, 2000, p. 42), refiriéndose expresamente a la teoría más fructífera, es decir, la teoría de sistemas. Su consecuencia es dejar atrás en su teoría de sistemas los conceptos de humano, individuo, sujeto, acción, a favor del concepto de comunicación.
} 
como el trabajo (Leistung) de orientarse (zurückzufinden) en una situación para vislumbrar (ausmachen) posibilidades de acción a través de las cuales la situación sea manejable ${ }^{27}$ (Stegmaier, 2014, p. 268). En situaciones que en principio son poco claras, nunca totalmente accesibles, y por eso permanecen inciertas, la orientación proporciona tanta certeza que se cree poder actuar (lo cual incluye también formular teorías) y, sin embargo, siempre se mantiene abierto decidir de otro modo en razón de nuevos indicios. La orientación no se basa en una teoría (tampoco en una filosofía de la orientación), pero puede valerse de teorías. Por el contrario, las decisiones teóricas siempre suponen orientaciones que aquellas ya no pueden alcanzar mediante teorías, pues también éstas presupondrían de nuevo orientaciones.

La orientación está siempre vinculada a puntos de vista, horizontes y perspectivas. Siempre es orientación en [in] una situación acerca de esta situación, es decir, es autoreferencial o autológica: obtiene sus resultados dependiendo de sí misma. En el miedo, por ejemplo, uno se orienta de modo diferente que en la calma. La orientación es recursiva o autopoiética: toma la orientación alcanzada como punto de partida para una nueva orientación, se reproduce a sí misma continuamente; incluso una situación sobre la que uno se ha orientado es una situación nueva, la que otra vez requiere de nueva orientación. Se tiene que partir igualmente, como Luhmann en su teoría de sistemas, de una diferencia entre orientación y situación. Esta es además una diferencia entre sistema y entorno, y también la orientación es un sistema de observación. Sin embargo, ésta no es sencillamente la orientación de hombres individuales, pues los que se orientan no son simplemente hombres que se orientan y en absoluto conscientemente: la orientación puede operar conscientemente, psíquicamente, pero asimismo (y empíricamente, en gran medida) física e inconscientemente. También las plantas y los animales se orientan y, en muchos aspectos, mejor que los hombres. Ella también incluye sistemas sociales (evidentemente también en animales), pero tiene un modo muy propio de lograrse y de fallar: por ejemplo, tal como ya lo constató Kant

Se puede proceder así, pero entonces obviamente la teoría misma se torna un problema. Para plantearla, por su parte, como problema, recurrimos al concepto de orientación.

${ }^{27}$ También para Luhmann el "problema del orientar-se (Sich-Zurechtfinden) en un mundo ante todo sin sentido, que está poblado por otros seres humanos errantes, igualmente desorientados" era lo "más originario". Él abordó este problema en conexión con Talcott Parsons, con el teorema de la doble contingencia, que aquí debemos dejar de lado. Cfr. para lo que sigue también Werner Stegmaier (2011a, pp. 63-73; 2015 y 2021). 
(1997, AA IV, p. 484), uno no puede percibir, definir ni observar-como se diría con el concepto de Luhmann- derecha e izquierda; una diferenciación que está en juego en cualquier orientación y, sin embargo, puede uno orientarse hacia allá y ocasionalmente también equivocarse (uno puede ver hacia la derecha, pero no puede ver allí la derecha). De esta manera, como sistema de observación, la orientación es igualmente autónoma. En su constitución autológica, autopoiética, autónoma, ella puede describirse bien con ayuda de la teoría de sistemas de Luhmann. Y, a la inversa, se pueden entender los sistemas de la teoría de sistemas como sistemas de orientación.

La orientación no sólo es primaria, porque toda observación inevitablemente comienza con ella, sino también singular, no generalizable. Ninguna orientación puede ocupar o 'compartir' el punto de vista de otra, no sólo local, sino también 'mentalmente', en tanto que, como resulta común, entre humanos pertenece al punto de vista aun todo lo que determina sus concepciones y opiniones (incluso en la ciencia cada uno tiene su punto de vista), al fin y al cabo toda su historia de vida y formación, que sólo es la suya. Como singulares, también las orientaciones mentales son y permanecen separadas unas de otras: no sólo no se puede mirar en 'el interior' de otros, saber lo que piensan en lo que hacen o dicen, sino que aun cuando uno cree entenderse recíprocamente bien, esto no es verificable, sea en palabras o signos que de nuevo otros pueden entender de manera diferente; los horizontes de orientación no se dejan 'fusionar' en el sentido de Gadamer (Gadamer, 1960, pp. 289ss.). La orientación singular es, pues, solitaria: está sola consigo misma, siempre puede orientarse respecto a otras orientaciones solamente en la suya propia.

Precisamente por esto la orientación es la más profunda necesidad: obtener apoyo en otro y otros no obstante la incertidumbre y el aislamiento de la propia situación. Sin orientación nada es posible, pues la desorientación es insoportable, pues más aún que el dolor, persiste, se acrecienta hasta el miedo y la desesperación. Cuando gana terreno una desorientación en alto grado, como en la 'muerte de Dios', la antropología puede tornarse una necesidad de orientación, tal como se esbozó junto a los dilemas ya descritos a los que ella conlleva. Entran aquí de nuevo en escena los hombres, no tanto en la forma de un concepto general y universalmente válido de 'hombre', sino como otras orientaciones humanas. Es evidente que mayoritariamente las orientaciones humanas se apoyan recíprocamente en la orientación-así como también pueden desorientarse unas a otras del modo más amenazante- y 
también reaccionan unas contra otras con la mayor fuerza. En esto se perciben recíprocamente como humanos concretos, completos, individuales. Por lo general, los humanos concretos, completos, individuales, no pasan inadvertidos en una situación, y su aparición puede de forma inmediata modificar totalmente la orientación, pues, finalmente, para bien o para mal, la orientación humana depende normalmente de hombres individuales y concretos. El lenguaje y la escritura, tal como Luhmann sostuvo, no son ante todo 'medios de comunicación simbólicamente generalizados', tal como él los denominó, y que inmediatamente fascinan, estimulan y ocupan la orientación humana, ni tampoco personas en roles sociales conocidos, sino hombres individuales, 'aún no fijados', según la fórmula de Nietzsche, de los cuales aún no se sabe qué se puede esperar. Ellos exigen orientación rápida, alta atención, observación aguda. Esto lo muestra del modo más manifiesto el cara a cara, la situación llena de tensión entre hombres (por así llamarlos ahora nuevamente) ${ }^{28}$. Esta tensión se puede observar hasta en los complejos procedimientos de búsqueda y evitación de la mirada, arraigados inmemorialmente: se decide de manera veloz y apenas consciente, pero con amplias consecuencias para el trato mutuo en el futuro y aun en roles sociales definidos, si se 'podrá mutuamente' o no, y además 'si' y 'cómo' se abrirá para ello uno al otro. Así pues, dicho en lenguaje sociológico, se decide sobre inclusión y exclusión. Por lo demás, para la orientación humana nada es tan interesante como los otros hombres concretos, sus destinos e historias, sus singularidades y caracteres, sus intereses y motivos, sus expectativas y esperanzas. Ellos son objeto constante de nuevos diálogos, noticias, relatos, dramas, películas, que proveen con posibilidades de acción la orientación propia para situaciones (más o menos) comparables, y siempre son tratados como hombres concretos y completos. Es por ello que uno se formará ciertas representaciones y conceptos de estas otras personas, pero mantendrá flexibles sus conceptos para poder modificarlos frente a eventuales sorpresas y para no abstraerlos demasiado, porque de lo contrario aportan muy poco para el trato concreto con las personas respectivas. Tanto menos resisten la prueba los conceptos abstractos y fijos de 'hombre en general', si uno no quiere ver cómo los hombres individuales en situaciones individuales terminan desviándose de ellos.

\footnotetext{
${ }^{28}$ Fue Emmanuel Levinas quien puso el face-à-face en el centro de la pregunta por el hombre, y extrajo radicalmente de allí nuevas consecuencias para la ética. Las antropologías humanistas, por el contrario, parten de hombres ya distendidos, prudentes, desprovistos de todo interés y pasión, completamente entregados a la razón, quienes luego con tanta más pasión deben desarrollar mutuamente un interés 'noble, beneficioso y bueno'.
} 
Sin embargo, más que orientarse acerca de [über] otros, uno se orienta en $[a n] \operatorname{otros}^{29^{*}}-\mathrm{y}$ la mayoría de las veces imperceptiblemente. La orientación en (...) es un modo de orientación específico, su tipo característico. Este modo predomina en ella, particularmente en lo que respecta a otros hombres, y no es alcanzable por la teoría. Orientación en (...) significa orientación en puntos de referencia. Cada orientación busca y elige sus puntos de referencia en su situación, según sus necesidades y en virtud de sus experiencias. Sin embargo, en la incertidumbre de la situación ella se atiene a éstos sólo provisionalmente, es decir, con reserva y transitoriamente, para buscar otros puntos de referencia adecuados, que la apoyen para actuar a pesar de la incertidumbre. La orientación en (...) diferencia constantemente puntos de referencia, decide su durabilidad y de esta manera mantiene siempre abiertos márgenes de acción para probar alternativas cuando surgen nuevos puntos de referencia. Ella decide bajo una incertidumbre permanente, que se reajusta siempre de nuevo; opera, experimenta en el mismo sentido en la dirección que Nietzsche y Luhmann indicaron, y puede, cuanto mejor resultado produzca, experimentar la incertidumbre no sólo como necesidad, sino también como felicidad. Alegra y a veces enorgullece poder orientarse bien.

También cuando uno se orienta acerca de otros hombres, sucede esto en gran medida en $[a n]$ puntos de referencia que son muy heterogéneos y pueden permanecer latentes, y rara vez sobre conceptos explícitos y consistentes. Cuando termina provisionalmente la orientación acerca de (...) se decide si se llega a un nuevo modo de orientación en (...); orientación, pues, en los hombres, sobre los cuales uno se ha orientado de momento. Pues como conjunto, los hombres se convierten en puntos de referencia de la orientación propia; uno se adhiere a ellos en lo que dice, hace, piensa, pero tampoco de manera incondicional, sino siempre dentro de márgenes de acción, porque las orientaciones están separadas y las situaciones son diferentes y, entonces, si se produce el 'acaso', no sucede simultáneamente en todos los aspectos: puede ser un camino que alguien toma, y que uno sigue porque no se conoce a sí mismo, o bien una indicación o un consejo que se pide a alguien, o bien el vestido y la conducta con los que también uno podría sentirse bien, o la actitud moral con la que alguien marca una señal, etc. Se creció así, con la

\footnotetext{
29 *Nota del traductor: el autor distingue aquí dos usos reflexivos del verbo orientieren [orientar] según la preposición que lo acompaña: sich orientieren über (orientarse sobre): informarse de, ponerse al corriente. Sich orientieren an [orientarse en]: mirarse en algo o alguien; guiarse por.
} 
orientación en [an] otros -padres, maestros, amigos, grupos, ídolos. Luego, en la pubertad, se logra una orientación intelectual acentuadamente propia, pero en la mayoría de asuntos se presenta también una orientación en otros, aunque ahora más firmemente con observaciones y decisiones propias acerca de 'a quién' preferiblemente se puede y quiere seguir. Esto ocurre también en gran parte de modo latente, rutinariamente, mucho más que a través del convencimiento consciente de las normas e ideales de orden social, en los que uno se inserta de esta manera. Definitivamente, las decisiones propias de orientación (por ejemplo, fundar una empresa, comprometerse políticamente para cambiar ordenamientos sociales o para salir de ellos) o tales como aquellas que se considera son, por el contrario, comparativamente riesgosas, requieren, si han de abrir posibilidades prometedoras de orientación, particular cautela y reflexión, y por ello son más bien raras -y tanto más se comunican y discuten. Tales decisiones saltan a la vista más intensamente, mientras la orientación en (an) otros pasa por normal, ya que aquí también se ofrecen por demás decisiones de orientación que otros ya han encontrado y que se pueden adoptar dentro de los propios márgenes de acción.

Así sucede también en orientaciones separadas que se presentan en segmentos muy extensos de comportamiento colectivo y de consenso, sin que para ello se tenga necesidad de especiales esfuerzos conceptuales. Sin embargo, siempre quedan márgenes para comportamiento alternativo, que luego puede ser discriminado moralmente como divergente, pero también puede ser apreciado económica, política o artísticamente como creativo. La orientación tampoco se deja limitar mediante delimitaciones conceptuales, también contra éstas se mantienen abiertos márgenes de maniobra en [an] los que uno también se orienta, porque siempre se debe evaluar primero hasta qué punto son ellos de importancia en la respectiva situación, si ellos son aquí los 'correctos' y, de ser así, qué peso se les debe otorgar, qué tan seriamente se los debe tomar. También deben ser reservados márgenes de orientación como márgenes de decisión contra ellos, también contra leyes y normas, ideales y teorías, pues de lo contrario uno no podría desenvolverse.

Y cuando uno se orienta en conceptos, leyes, normas, teorías ideales, que sobrepasan una cierta complejidad, pero sobre todo cuando se tiene que ver con organizaciones que a partir de cierto tamaño se vuelven complejas para un observador externo, tanto más se atiene uno de nuevo a los hombres individuales. Estos pueden convertirse entonces en 'rostros' de aquellos, 'modelos' de la observancia de normas y leyes, y de la vida según ideales 
(o también en ejemplos de su desacato), en 'representantes' de teorías, en 'rótulos' de organizaciones, en 'símbolos' de pueblos y Estados, y hasta en 'signos' de una época $y$, de momento, aun ante cualquier pregunta de poder y rango, resultan entonces decisivos respecto a saber si y cómo uno se comporta frente a aquello de lo que se trata. Una médica amigable y preocupada puede hacer soportable un hospital, que de lo contrario sería insoportable, y un médico fraudulento puede desacreditar todo el sistema hospitalario. Números, como los de las víctimas en una guerra civil, sólo 'dicen' algo cuando ellos hacen ver a los hombres concretos y sus destinos. Los individuos son el problema más grande de la orientación humana, pero también los puntos de referencia más indispensables y útiles para todo lo demás. En lugar de antropologías, en apariencia doctrinas universales del hombre en general, se tiene que comenzar, entonces, con la orientación en la orientación humana, que finalmente es siempre la de hombres individuales en otros hombres individuales, a los cuales siempre, incluso contra todas las teorías y doctrinas, les quedan márgenes de decisión. Una filosofía de la orientación va más allá de una antropología que la puede fácilmente distorsionar.

\section{Referencias}

Aristoteles (1998). Politik. Schwarz, F. (Hrsg.). Leipzig: Reclam.

Bertino, A. C. (2011). ,,Vernatürlichung“. Ursprünge von Friedrich Nietzsches Entidealisierung des Menschen, seiner Sprache und seiner Geschichte bei Johann Gottfried Herder. Berlin / Boston: Walter de Gruyter.

Constâncio, J. and Mayer, M. (Eds.). (2011). Nietzsche on Instinct and Language. Nietzsche Today. Berlin / Boston: Walter de Gruyter.

Dziewas, R. (1992). Der Mensch - ein Konglomerat autopoietischer Systeme? Krawietz, W. und Welker, M. (Hrsg.). Kritik der Theorie sozialer Systeme. Auseinandersetzungen mit Luhmanns Hauptwerk, (pp. 113132). Frankfurt a. M.: Suhrkamp.

Erb, M. (2010). Evolution, Genealogie und ,Gegen-Anthropologie'. Michel Foucaults Frühe Auseinandersetzung mit Darwin und Nietzsche. Reschke, R. und Gerhardt, V. (Hrsg.). Nietzsche-Forschung, 17 (1), pp. 137-148. 
Figl, J. (1982). Interpretation als philosophisches Prinzip. Friedrich Nietzsches universale Theorie der Auslegung im späten Nachlaß. Monographien und Texte zur Nietzsche-Forschung. Berlin / New York: Walter de Gruyter.

Fischer, J. (2008). Philosophische Anthropologie. Eine Denkrichtung des 20. Jahrhunderts. Freiburg / München: Karl Alber.

Foucault, M. (1961). Wahnsinn und Gesellschaft. Eine Geschichte des Wahns im Zeitalter der Vernunft. (Trad. Köppen, U.). Frankfurt a. M.: Suhrkamp.

Foucault, M. (2003). Die Ordnung der Dinge: Eine Archäologie der Humanwissenschaften. (Trad. Köppen, U.). Frankfurt a. M.: Suhrkamp.

Gadamer, H. G. (1960). Wahrheit und Methode. Grundzüge einer philosophischen Hermeneutik. Tübingen: J. C. B. Mohr.

Gehlen, A. (1971). Der Mensch, seine Natur und seine Stellung in der Welt (1940). Frankfurt a. M.: Athenäum.

Geisenhanslücke, A. (1999). Der Mensch als Eintagswesen. Nietzsches kritische Anthropologie in der Zweiten Unzeitgemäßen Betrachtung. Nietzsche-Studien, 28 (1), pp.125-140.

Gillespie, M, A. (1999). Nietzsche and the Anthropology of Nihilism. Nietzsche-Studien, 28 (1), pp. 141-155.

Heidegger, M. (1929). Kant und das Problem der Metaphysik. Bonn: Cohen.

Heimsoeth, H. (1943). Zur Anthropologie Friedrich Nietzsches. Berlin: Junker und Dünnhaupt Verlag.

Hoffmann, Th. S.; Rolke, L. und Gosepath, S. (1992). Rationalität, Rationalisierung. Ritter, J. et al. (Hrsg.). Historisches Wörterbuch der Philosophie (B. 8), (pp. 52-66). Basel / Darmstadt: Schwabe \& Co.

Kant, I. (1962). Logik. Weischedel, W. (Hrsg.). Darmstadt: Wissenschaftliche Buchgesellschaft.

Kant, I. (1997). Metaphysische Anfangsgründe der Naturwissenschaft. (Übers. Pollock. K). Leipzig: Meiner. 
Lemm, V. (2009). Nietzsche's Animal Philosophy. Culture, Politics, and the Animality of the Human Being. New York: Fordham University Press.

Loukidelis, N. (2012). Anthropologie bei Nietzsche und Gehlen. Mit einem Blick auf Trendelenburg. Reschke, R. und Brusotti, M. (Hrsg.). „Einige werden posthum geboren". Friedrich Nietzsches Wirkungen, (pp. 117127). Berlin / Boston: Walter de Gruyter.

Luhmann, N. (1978). Soziologie der Moral. Stephan, H. (Hrsg.). Theorietechnik und Moral, (pp. 8-116). Frankfurt a. M.: Suhrkamp Verlag.

Luhmann, N. (1980). Frühneuzeitliche Anthropologie. Theorietechnische Lösungen für ein Evolutionsproblem der Gesellschaft. Gesellschaftsstruktur und Semantik: Studien zur Wissenssoziologie der modernen Gesellschaft. Frankfurt a. M.: Suhrkamp.

Luhmann, N. (1984). Soziale Systeme. Grundriß einer allgemeinen Theorie. Frankfurt a. M.: Suhrkamp.

Luhmann, N. (1995a). Wie ist Bewußtsein an Kommunikation beteiligt? Soziologische Aufklärung: Die Soziologie und der Mensch, (pp. 37-54). Wiesbaden: Sozialwissenschaften Verlag.

Luhmann, N. (1995b). Die Soziologie und der Mensch. Soziologische Aufklärung: Die Soziologie und der Mensch, (p. 265-274). Opladen: Westdeutscher Verlag.

Luhmann, N. (2000). Was ist Kommunikation? Vortrag auf dem Symposium „Lebende Systeme. Konstruktion und Veränderung von Wirklichkeiten und ihre Relevanz für die systemische Therapie“. Gente, P.; Paris, H. und Weinmann, M. (Hrsg.). (pp. 41-63). Frankfurt a. M.: Grin Verlag.

Luhmann, N. (2008). Die Moral der Gesellschaft. Horster, D. (Hrsg.). Frankfurt a. M.: Suhrkamp.

Luhmann, N. (2011). Organisation und Entscheidung. Wiesbaden: Sozialwissenschaften Verlag.

Marquard, O. (1971). Anthropologie. Ritter, J. et al. (Hrsg.). Historisches Wörterbuch der Philosophie (B. 1), (pp. 362-367). Basilea / Darmstadt: Schwabe \& Co. 
Meckel, M. (1980). Der Weg Zarathustras als der Weg des Menschen. Zur Anthropologie Nietzsches im Kontext der Rede von Gott im „Zarathustra”. Nietzsche-Studien, 9 (1), pp. 174-208.

Müller-Lauter, W. (1984). Nihilismus I. Ritter, J. et al. (Hrsg.). Historisches Wörterbuch der Philosophie (B. 6), (pp. 846-853). Basilea / Darmstadt: Schwabe \& Co.

Nietzsche. F. (1882). Diefröhliche Wissenschaft. Chemnitz: Ernst Schmeitzner.

Nietzsche. F. (1887). Zur Genealogie der Moral. Leipzig: C. G. Neumann Verlag.

Nietzsche, F. (1988). Die Geburt der Tragödie. Unzeitgemäße Betrachtungen. Nachgelassene Schriften 1870-1873 (KSA 1). Kritische Studien Ausgabe. Colli, G. und Montinari, M. (Hrsg.). Berlin: Walter de Gruyter.

Nietzsche, F. (1988) Also sprach Zarathustra (KSA 4). Kritische Studien Ausgabe. Colli, G. und Montinari, M. (Hrsg.). Berlin: Walter de Gruyter

Nietzsche, F. (1988). Nachlas 1872-1873 (KSA 7). Kritische Studien Ausgabe. Colli G. und Montinari, M. (Hrsg.). Berlin: Walter de Gruyter.

Nietzsche, F. (1988). Nachgelassene Fragmente 1880-1882 (KSA 9). Kritische Studien Ausgabe. Colli, G. und Montinari, M. (Hrsg.). Berlin: Walter de Gruyter.

Nietzsche, F. (1988). Nachgelassene Fragmente 1882-1884 (KSA 10). Kritische Studien Ausgabe. Colli, G. und Montinari, M. (Hrsg.). Berlin: Walter de Gruyter.

Nietzsche, F. (1889). Götzen-Dämmerung oder: Wie man mit dem Hammer philosophiert. Leipzig: C. G. Neumann Verlag.

Nietzsche, F. (2015). Über Wahrheit und Lüge im außermoralischen Sinne. Sina, K (Hrsg). Stuttgart: Reclam.

Nietzsche, F. (2016). Der Antichrist. Fluch auf das Christenthum. Berlin: Holzinger.

Nietzsche. F. (2018). Jenseits von Gut und Böse. Tegernsee: Boer Verlag.

Rölli, M. (2005). Anthropologie im Widerstreit. Eine Konfrontation von Kant und Nietzsche. Himmelmann, B. (Hrsg.). Kant und Nietzsche im Widerstreit, (pp. 346-359). Berlin / NewYork: Walter de Gruyter. 
Schacht, R. (2005). Kant, Nietzsche und „der Mensch“. Die Idee und Aufgabe einer Philosophischen Anthropologie. Himmelmann, B. (Hrsg.). Kant und Nietzsche im Widerstreit, (pp. 277-294). Berlin / New York: Walter de Gruyter.

Schacht, R. (2006). Nietzsche and Philosophical Anthropology. Pearson K. A. (Ed.). A Companion to Nietzsche, (pp. 115-132). Oxford: Wiley Balckwell.

Schiller, F. (Hrsg.). (1797). Tabulae votivae. Musen-Almanach für das Jahr 1797, (p. 152-182). Tübingen: Cotta.

Schimank, Uwe. (2005). „Gespielter Konsens“: Fluchtburg des Menschen in Luhmanns Sozialtheorie. Runkel, G. und Burkart, G. (Hrsg.). Funktionssysteme der Gesellschaft. Beiträge zur Systemtheorie von Niklas Luhmann, (pp. 265-284). Wiesbaden: Sozialwissenschaften Verlag.

Schipperges, H. (1975). Am Leitfaden des Leibes. Zur Anthropologie und Therapeutik Friedrich Nietzsches. Stuttgart: Klett Verlag.

Schlossberger, M. (1998). Über Nietzsche und die Philosophische Anthropologie. Nietzsche-Forschung, 4 (1), pp. 147-167.

Skowron, M. (2013). Posthuman oder Übermensch. War Nietzsce ein Transhumanist? Nietzsche-Studien, 42 (1), pp. 256-282.

Stegmaier, W. (1987). Darwin, Darwinismus, Nietzsche. Zum Problem der Evolution. Nietzsche-Studien, 16 (1), pp. 264-287.

Stegmaier, W. (1995). Philosophieren als Vermeiden einer Lehre. Interindividuelle Orientierung bei Sokrates und Platon, Nietzsche und Derrida. Simon, J. (Hrsg.). Distanz uns Verstehen, (pp. 214-239). Frankfurt a. M.: Suhrkamp.

Stegmaier, W. (2000). Anti-Lehren. Szene und Lehre in Friedrich Nietzsches Also sprach Zarathustra. Gerhardt, V. (Hrsg.). Friedrich Nietzsche, Also sprach Zarathustra, (pp. 191-224). Berlin: Walter de Gruyter.

Stegmaier, W. (2008). Philosophie der Orientierung. Berlin / New York: Walter de Gruyter. 
Stegmaier, W. (2010). Der See des Menschen, das Meer des Übermenschen und der Brunnen des Geistes. Fluss und Fassung einer Metapher Friedrich Nietzsches. Nietzsche-Studien, 39 (1), pp. 145-179.

Stegmaier, W. (2011a). Der Mensch als Orientierungswesen. Anthropologie zwischen Alter und ego. Dalferth, I. und Hunziker, A. (Hrsg.). Seinkönnen. Der Mensch zwischen Möglichkeit und Wirklichkeit, (pp. 63-73). Tübingen: Mohr Siebeck.

Stegmaier, W. (2011b). Nietzsche zur Einführung. Hamburg: Junius Verlag.

Stegmaier, W. (2014). Philosophie der Orientierung. Berlin / New York: Walter de Gruyter.

Stegmaier, W. (2015). Subjects as temporal clues to orientation: Nietzsche and Luhmann on Subjectivity. Constâncio, J. (Ed). Nietzsche and the Problem of Subjectivity, (pp. 63-73). Berlin / Boston: Walter de Gruyter.

Stegmaier, W, (2021). Orientierung am Andern. Rocha de la Torre, A. (Hrsg.). Die Frage nach dem Anderen in der gegenwärtigen Philosophie (Proceso de edición).

Visser, G. (1999). Nietzsches Übermensch. Die Notwendigkeit einer Neubesinnung auf die Frage nach dem Menschen. Nietzsche-Studien, 28 (1), pp. 100-124. 